\title{
Preparation and Some Properties of Active Protoplasts of Bacillus megaterium
}

\author{
By E. CUNDLIFFE \\ Subdepartment of Chemical Microbiology, Department of Biochemistry, \\ University of Cambridge
}

(Accepted for publication 17 May 1968)

\begin{abstract}
SUMMARY
A method for the preparation of protoplasts of Bacillus megaterium is described. The protoplasts obtained were very active metabolically and lysates of them were rich in polyribosomes. Methods for the detection of polyribosomes and of nascent peptides are detailed.
\end{abstract}

\section{INTRODUCTION}

A protoplast may be defined as 'a structure derived from a vegetative cell by removal of the entire cell wall, or alternatively that part of the cell which lies within the cell wall and which, in some species, may be plasmolysed away from it' (McQuillen, 1960). It is a consequence of the general difference between the cell walls of Gram-positive and Gram-negative organisms that true protoplasts can only, at present, be obtained from certain Gram-positive species. The methods used to prepare protoplasts and a description of their properties are given in the review of McQuillen (I960). The present method for the preparation of protoplasts was devised so that studies of protein synthesis in Bacillus megaterium could be extended to include investigations of the effects of antibiotics upon polyribosomes, which can be obtained from protoplasts by gentle methods (i.e. methods which involve little or no mechanical shearing which would be expected to break polyribosomes). It was also felt that these studies would be more significant if the protoplasts could be obtained in a reproducible vigorously active metabolic state, so that they could be compared with bacteria in the logarithmic phase of growth. Therefore a rapid method for the quantitative conversion of $B$. megaterium to protoplasts was devised; the method is basically similar to that of Yudkin (1963).

\section{METHODS}

Cultivation. Lyophilized cultures of Bacillus megaterium KM were used to start new stocks at monthly intervals. The organism was maintained by subculturing daily in a liquid medium C (McQuillen \& Roberts, 1954) with 0.I \% (w/v) glucose added. For experiments, a basal liquid medium PRE was used. This was similar to medium PR of Yudkin (1963) and contained (g./1.): $\mathrm{KCl}, 4 ; \mathrm{MgCl}_{2} .6 \mathrm{H}_{2} \mathrm{O}, 4 ; \mathrm{NH}_{4} \mathrm{Cl}, 2 ; \mathrm{Na}_{2} \mathrm{SO}_{4}$, $0.15 ; \mathrm{Na}_{2} \mathrm{HPO}_{4}$. $\mathrm{I}_{2} \mathrm{H}_{2} \mathrm{O}, 0.35$; glucose, I0; sucrose, 100. Difco Bacto-peptone (0.1 \%, $\mathrm{w} / \mathrm{v}$ ) was added when required. Organisms were grown in the basal medium supplemented with peptone at $37^{\circ}$ with aeration in the presence of ${ }^{32} \mathrm{P}$-orthophosphate ( $15 \mu \mathrm{c} / \mathrm{ml}$.) for three generations. Under these conditions the extinction at $600 \mathrm{~m} \mu$ $\left(E_{600}\right)$ doubled in $30-35 \mathrm{~min}$. 
Preparation of protoplasts. Organisms in the exponential phase of growth, at suspension densities of about $200 \mu \mathrm{g}$. dry weight $/ \mathrm{ml}$., were harvested by centrifugation ( $3 \mathrm{~min}$. at $3000 \mathrm{~g}$ ) and resuspended at $10 \mathrm{mg} . / \mathrm{ml}$. in the basal medium containing peptone and lysozyme $\left(200 \mu \mathrm{g} . / \mathrm{ml}\right.$.). At $37^{\circ}$ the bacilli were quantitatively converted to protoplasts within 2-3 min. It was important that the organisms should be in the logarithmic phase of growth at the time of harvesting, and that harvesting and resuspension were carried out rapidly, otherwise conversion to protoplasts was poor. However, there was no absolute dependence on growth rate. Organisms grown in the absence of peptone (doubling time about $70 \mathrm{~min}$.) were converted equally rapidly, provided they were harvested in the exponential phase of growth. The presence of peptone was not essential during the formation of protoplasts.

Incubation of protoplasts. Immediately after preparation, protoplast suspensions were diluted 50-fold with medium plus peptone (to $200 \mu \mathrm{g} . / \mathrm{ml}$.) and were incubated with shaking at $37^{\circ}$. Important variables here were the size and shape of the vessel used and the volume of suspension in it. Usually $10-15 \mathrm{ml}$. of protoplast suspension was incubated in a $100 \mathrm{ml}$. conical flask. During this incubation, the $E_{600}$ of the suspension initially decreased by about $10 \%$ (presumably due to osmotic imbalance across the membrane) and then began to increase exponentially. The time taken for the extinction to double was usually 25-30 min., slightly less than that for whole cells in the logarithmic phase of growth in the same medium. It was also observed that the distribution of polyribosomes in sucrose density gradients changed markedly during this incubation (see later). In no case did the apparent number of protoplasts increase even when the $E_{600}$ doubled; rather the protoplasts increased in size.

Lysis of protoplasts. Samples of protoplasts (usually $0.5 \mathrm{ml}$.) were pipetted as rapidly as possible, using pre-warmed pipettes, into thin-walled glass vials standing in a metal block in ice. Each vial contained the neutral detergent, Triton XIoo and deoxyribonuclease so that after addition of the sample the final concentrations were $0.1 \%(\mathrm{v} / \mathrm{v})$ and $5 \mu \mathrm{g} . / \mathrm{ml}$. respectively. In some experiments ribonuclease was also present in the vial (final concentration $5 \mu \mathrm{g}$. $/ \mathrm{ml}$.). After I min. in ice, the resultant lysates were fractionated at temperatures not exceeding $4^{\circ}$.

Sucrose density gradient analysis. Linear sucrose density gradients having a total volume of $4.6 \mathrm{ml}$. were prepared at $3^{\circ}$ and were allowed to stand at that temperature for $5^{-18} \mathrm{hr}$ before use. Each gradient ranged from $\mathrm{I} 5$ to $40 \%(\mathrm{w} / \mathrm{v})$ sucrose in a buffer containing tris, $0.01 \mathrm{M}$; magnesium acetate, $0.0 \mathrm{I} \mathrm{M} ; \mathrm{KCl}, 0 . \mathrm{I} \mathrm{M} ; \mathrm{pH} 7.5$ at $10^{\circ}$ adjusted with $\mathrm{HCl}, 0.2 \mathrm{ml}$. of protoplast lysate was layered on each gradient.

Centrifugation was carried out at $2^{\circ}$ for $40 \mathrm{~min}$. by using the Spinco SW 39 rotor at $38,000 \mathrm{rev} . / \mathrm{min}$. The centrifuge was evacuated for $5 \mathrm{~min}$. before each run. After spinning, the bottom of each tube was pierced with a needle, and successive fractions of 8 drops (about $0.13 \mathrm{ml}$. total) were collected into tubes containing I ml. cold $8 \%$ $(\mathrm{w} / \mathrm{v})$ trichloroacetic acid.

The trichloroacetic acid-precipitates were collected on Oxoid membrane filters, rinsed once with $2 \mathrm{ml} .5 \%(\mathrm{w} / \mathrm{v})$ trichloroacetic acid and twice with $2 \mathrm{ml}$. I \% (v/v) acetic acid, dried at $80^{\circ}$ for $30 \mathrm{~min}$. and then counted in a liquid-scintillation counter (Nuclear Chicago Corporation). A toluene-based scintillation fluid containing $4 \mathrm{~g}$. of 2,5-bis-(5'-t-butylbenzoxazolyl-2')-thiophene (BBOT)/l. was used. Routinely $39 \pm$ I gradient fractions were collected. The distribution of ${ }^{32} \mathrm{P}$ from steady-state labelled systems was found to be an accurate indicator of the polyribosome profile and was far 
more sensitive than the alternative method of estimating extinction at $260 \mathrm{~m} \mu$. Sedimentation coefficients of materials running in the gradients were not directly measured. The term $70 \mathrm{~S}$ has been used to describe material sedimenting in the same region as the major peak in ribonuclease-treated lysates.

\section{MATERIALS}

Deoxyribonuclease (electrophoretically purified) was obtained from the Worthington Biochemical Corporation, Freehold, N.J., U.S.A. Pancreatic ribonuclease (Worthington) was held at $80^{\circ}$ for Io min. before use. Egg-white lysozyme was obtained from Armour Pharmaceutical Co., Ltd., Eastbourne, England. BBOT was obtained from Ciba Ltd. Triton XIoo was obtained from The Rohm \& Haas Co., Philadelphia, Pa., U.S.A. Actinomycin D (Dactinomycin) was a generous gift from Merck Sharpe and Dohme. Inc., Rahway, N.J., U.S.A. [ ${ }^{32}$ P] orthophosphate and $\left[{ }^{3} \mathrm{H}\right]$-L-phenylalanine $(5 \mathrm{C} / \mathrm{m}$-mole) were obtained from the Radiochemical Centre, Amersham, England.

\section{RESULTS}

A suspension of protoplasts was sampled immediately after preparation and later after incubation at $37^{\circ}$ for $20 \mathrm{~min}$. by which time the $E_{600}$ was increasing exponentially. Gradient analysis revealed that, typically, about $50 \%$ of the total ribosomal material was present as $70 \mathrm{~S}$ monomers initially but that during the incubation there was a

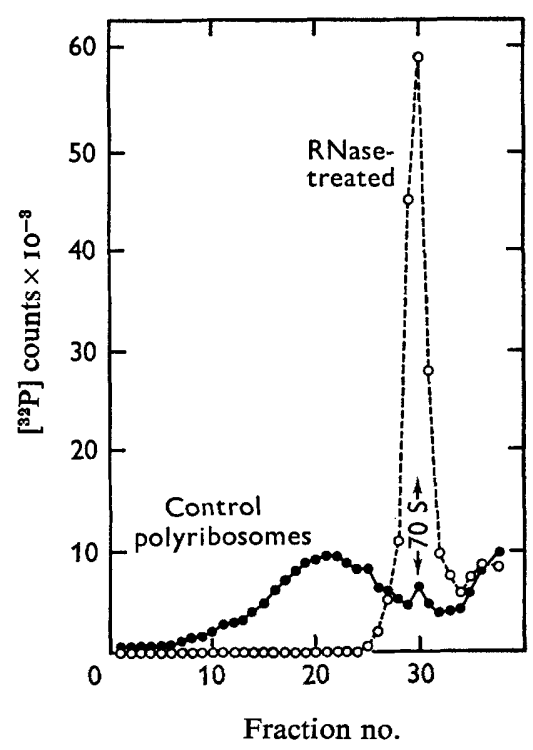

Fig. I. Polyribosomes from exponentially growing protoplasts: the effect of ribonuclease. [ $\left.{ }^{32} \mathrm{P}\right]-L a b e l l e d$ protoplasts were lysed during exponential growth. (0) A portion of the lysate was analysed on a sucrose density gradient. $(O)$ Another portion of the same lysate was treated with ribonuclease $\left(5 \mu \mathrm{g} . / \mathrm{ml}\right.$.) for $5 \mathrm{~min}$. at $0^{\circ}$ prior to gradient analysis.

decrease in the amount of $70 \mathrm{~S}$ material and a corresponding increase in heavier material (polyribosomes). Finally about $85-90 \%$ of the ribosomes were reproducibly recovered as polyribosomes (Fig. I). 
Protoplasts were routinely incubated in this manner prior to commencing each of the following experiments.

Effect of ribonuclease on polyribosomes. Protoplasts were sampled and the lysate was divided into two portions, one of which was treated with ribonuclease as described under Methods. This mild treatment with ribonuclease completely removed all

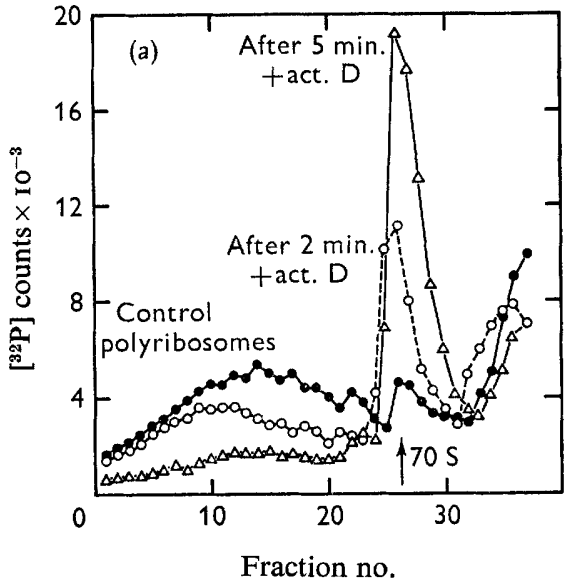

Fig. $2 a$

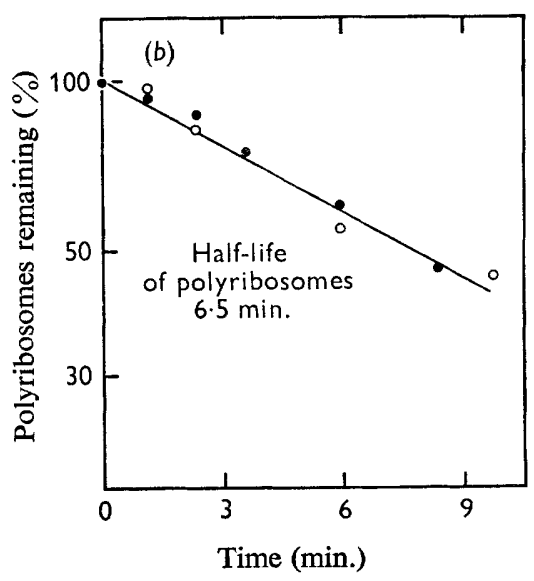

Fig. $2 b$

Fig. 2a. The effect of actinomycin D on polyribosomes. Actinomycin D (Io $\mu \mathrm{g} . / \mathrm{ml}$.) was added to a suspension of $\left[{ }^{32} \mathrm{P}\right]$-labelled protoplasts in the exponential phase of growth and the incubation was continued. Samples were taken at intervals and the subsequent lysates were analysed on sucrose density gradients. (๑) The protoplasts were sampled immediately before the addition of actinomycin. Other samples were taken $2 \mathrm{~min}$. (O) and $5 \mathrm{~min}(\triangle)$ after the addition of actinomycin.

Fig. $2 b$. Rate of breakdown of polyribosomes in the presence of actinomycin D. Data from duplicate experiments identical to that described in Fig. $2 a$. Percentages of polyribosomes remaining were computed as described in the text.

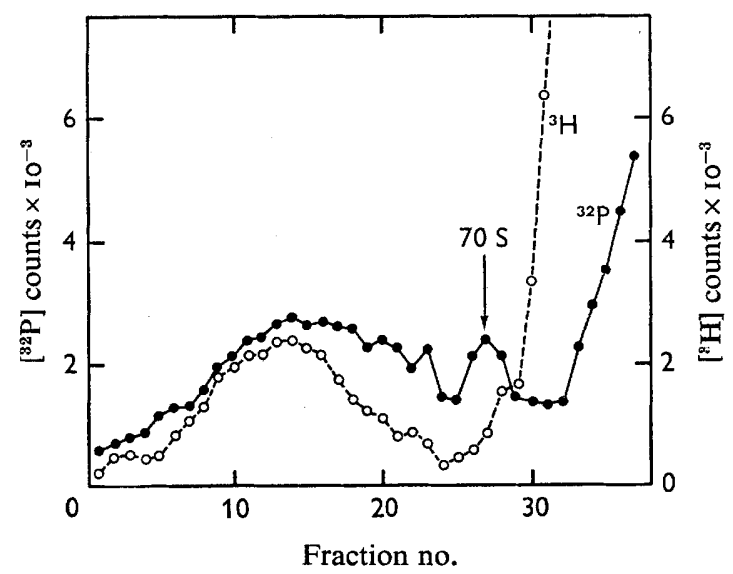

Fig. 3. Nascent protein and polyribosomes. $\left.{ }^{32} \mathrm{P}\right]$-Labelled protoplasts were incubated into the phase of exponential growth then $\left[{ }^{3} \mathrm{H}\right]$ phenylalanine $(5 \mathrm{c} / \mathrm{m}$-mole) was added (10 $\mu \mathrm{c}$. $\mathrm{ml}$.) for $60 \mathrm{sec}$. Then the protoplasts were sampled and the lysate analysed on a sucrose density gradient $(\odot)\left[{ }^{32} \mathrm{P}\right]$-Labelled polyribosomes, ribosomes, etc. (O) $\left[{ }^{3} \mathrm{H}\right]$-Labelled peptides. 
the polyribosomes giving a massive peak of monomeric ribosomes (Fig. I). The extreme sensitivity of the polyribosomes to ribonuclease is in accordance with current ideas concerning the structure of polyribosomes. Also it is obvious from Fig. I that some of these structures were very heavy and by assuming that $100 \%$ of the ribosomes present in the $70 \mathrm{~S}$ peak after ribonuclease treatment, it was calculated that at least $85 \%$ of the ribosomes had been present as polyribosomes. This is a greater proportion than is usually found in bacterial lysates (see however Flessel, Ralph \& Rich, 1967).

Effect of actinomycin $D$ on polyribosomes. Protoplasts were sampled and then actinomycin D (Io $\mu \mathrm{g}$. $/ \mathrm{ml}$.) was added to the remainder, incubation was continued and samples were taken at various times. Actinomycin caused a breakdown of polyribosomes with accumulation of ribosomes (Fig. 2a). The percentage of ribosomes present as polyribosomes was calculated for each time point as described above and the numbers obtained were expressed relative to the initial value. Figure $2(b)$ shows that the decay of polyribosomes exhibited approximately exponential characteristics with a half-life of about $6.5 \mathrm{~min}$.

Nascent protein and polyribosomes. Protoplasts were incubated with $\left[{ }^{3} \mathrm{H}\right]$ phenylalanine (Io $\mu \mathrm{c} / \mathrm{ml}$.) for $60 \mathrm{sec}$. and a sample was taken for lysis and gradient analysis. Figure 3 shows that $\left[{ }^{3} \mathrm{H}\right]$-labelled-material was recovered in the polyribosome region and at the top of the gradient in the 'soluble' region. The specific activity $\left[{ }^{3} \mathrm{H}\right] /\left[{ }^{32} \mathrm{P}\right]$ was greatest in the region where the large polyribosomes sedimented, with a minimum at $70 \mathrm{~S}$. This result is in agreement with the idea that nascent peptides are formed on polyribosomes and not on free $70 \mathrm{~S}$ ribosome-monomers.

\section{DISCUSSION}

A method was developed for the rapid and quantitative conversion of Bacillus megaterium to protoplasts. After incubation the protoplasts could be obtained in an active metabolic state and lysates of such protoplasts were rich in polyribosomes. Nascent protein was found to be associated with polyribosomes rather than with free $70 \mathrm{~S}$ monomers in keeping with current ideas concerning the mechanism of protein synthesis. Various methods have been developed whereby bacteria may be rendered sensitive to lysis by detergents or by osmotic shock (e.g. Schaechter, Previc \& Gillespie, 1965; Flessel et al. 1967; Mangiarotti \& Schlessinger, 1967). However, some of these methods involve the use of lysozyme, which does not convert drug-inhibited organisms to protoplasts and gentle lysis of such organisms is not usually possible.

Therefore it is considered that protoplasts offer an advantageous system for the study of protein synthesis in vivo since they can be lysed promptly and gently to yield undegraded polyribosomes still bearing nascent peptides. Studies of this kind involving antibiotics and protoplasts have been reported elsewhere (Cundliffe, 1967a, $b$; Cundliffe \& McQuillen, I967a,b).

The work described in this paper has been presented in abstract form (Cundliffe, 1967c) and as part of a dissertation (Cundliffe, 1967d).

I am grateful to the Medical Research Council for a scholarship for training in research methods and to Dr K. McQuillen for advice, criticism and encouragement. 


\section{REFERENCES}

CUNDLIFFE, E. (1967a). Chlortetracycline and polyribosomes. J. gen. Microbiol. 48, vi.

CUNDLIFFE, E. $(1967 b)$. Antibiotics and polyribosomes: chlortetracycline and polyribosomes of Bacillus megaterium. Mol. Pharmacol. 3, 401.

CunDLIFFe, E. (1967c). Polyribosomes in protoplasts of Bacillus megaterium.J. gen. Microbiol. 46, viii. CundLIFFe, E. (1967d). Ph.D. Dissertation, University of Cambridge, England.

Cundliffe, E. \& MCQuillen, K. (1967a). Peptide bond formation in bacteria. J. gen. Microbiol. 50, $x$.

Cundliffe, E. \& MCQUillen, K. (1967b). Bacterial protein synthesis: The effects of antibiotics. J. molec. Biol. 30, 137.

Flessel, C. P., RalPh, P. \& Rich, A. (1967). Polyribosomes of growing bacteria. Science, N.Y. $158,658$.

McQunlen, K. (1960). In The Bacteria, Ed. by U. C. Gunsalus and R. Y. Stanier. Vol. I, p. 249. New York and London: Academic Press.

MCQUILLEN, K. \& RoBerTs, R. B. (1954). The utilization of acetate for synthesis in Escherichia coli. J. biol. Chem. 207, $8 \mathrm{I}$.

Mangiarotti, G. \& SChlessinger, D. (1967). Polyribosome metabolism in Escherichia coli. J. molec. Biol. 20, 123.

Schaechter, M., Previc, E. P. \& GillesPie, M. E. (I965). Messenger RNA and polyribosomes in Bacillus megaterium. J. molec. Biol. I2, II9.

YudkIN, M. D. (1963). The effect of penicillin, novobiocin, streptomycin and vancomycin on membrane synthesis by protoplasts of Bacillus megaterium. Biochem. J. 89, 290. 